Inc., C. Stiening Employee of: Kypha, Inc., J. Atkinson Consultant for: Kypha, Inc. Compliment Corporation, Gemini Therapeutics, Inc., Celldex Therapeutics, Clinical Pharmacy Services, CDMI, Omeros Corporation, Achillion Pharmaceuticals, Inc., True North Therapeutics, BioMarin Pharmaceutical, Inc., Annexon Biosciences, Inc., and AdMiRx, Inc.

DOI: 10.1136/annrheumdis-2018-eular.1843

\section{FRI0344 $\quad$ SERUM FREE LIGHT CHAINS AS A FLARE BIOMARKER IN SYSTEMIC LUPUS ERYTHEMATOSUS}

A.B. Rodriguez Cambron ${ }^{1}$, J. Jimenez Jimenez ${ }^{2}$, M.A. Blazquez Cañamero ${ }^{3}$, M. J. García De Yébenes ${ }^{4}$, F. Rey Pazos ${ }^{5}$, C. Macía Villa ${ }^{1}$, M. Alcalde Villar1, F. Cabero Del Pozo ${ }^{1}$, P. Collado Ramos ${ }^{1}$, E. Alvarez Andrés ${ }^{1}$, A. Cruz Valenciano ${ }^{1}$. ${ }^{1}$ Rheumatology, ${ }^{2}$ Immunology, Hospital Universitario Severo Ochoa;

${ }^{3}$ Rheumatology, Hospital Universitario Ramón y Cajal; ${ }^{4}$ Musculoskeletal Health Institute (InMusc); ${ }^{5}$ Rheumatology, Hospital Universitario Infanta Sofía, Madrid, Spain

Background: Complement levels are already known as biomarkers of flare in systemic lupus erythematosus (SLE); currently the usefulness of free light chains (FLC) in different autoimmune diseases in which the $B$ cell has a relevant pathogenic role, as in the case of SLE, is being investigated.

Objectives: To explore the usefulness of FLC determination as a flare biomarker in patients with SLE and to analyse possible discriminative differences between FLC and complement C3 and C4 levels.

Methods: We performed an unicentric prospective longitudinal study with the following inclusion criteria: age greater than 18 years old and fulfilment of ACR or SLICC criteria for the diagnosis of SLE. Exclusion criteria were non-SLE related haematological disease, severe infection and severe kidney disease (Crea>2 $\mathrm{mg} / \mathrm{dl}$ ) to avoid interferences with FLC clearance. SLE flare definition was based on the SFI index. Receiver operator curves (ROC) and calculation of the area under the curve (AUC) were used to compare the discriminative ability between FLC and C3-C4 levels.

Results: 46 patients were enrolled. For the present communication, only baseline data were analysed. 41 (91\%) patients were women. Most frequent clinical manifestations were haematological (83\%) and cutaneous (72\%). Laboratory findings were $98 \%$ positive ANA, $67 \%$ positive antiDNAdc, $54 \%$ decreased $\mathrm{C} 3 \%$ and $39 \%$ decreased C4. 6 (13\%) patients presented a SLE flare and their characteristics compared to non-flare patients were lower C3 levels (70 vs $95 ; p=0,017$ ) and C4 levels $(10$ vs $17 ; p=0,008)$ with higher concentrations of lambda light chains $(\lambda L C)$ ( 27 vs $19 ; p=0,028)$. In addition, flare patients had higher IgA levels ( 402 vs 250 ; $p=0,029)$, higher score in the global assessment of the doctor (4.3 vs 1.6; $p=0,0003$ ), longer time disease evolution ( 17.8 vs 14.3 years; $p=0,844$ ), higher values of SLEDAI $(2.7 \pm 2$ vs $1.4 \pm 1.3 ; p=0,083)$ and higher lymphopenia (982 vs 1474; $p=0,068$ ). We found no association between FLC levels and the presence of antiDNAdc. The correlation of FLC with the rest of activity biomarkers (C3, C4, SLEDAI, VAS) occurred in the expected way although the magnitude of the association was moderate, with a higher correlation between $\mathrm{C} 3$ and $\lambda$ LC. $\lambda$ LC were the only FLC that showed ability to discriminate flares (AUC 0.781 ), with sensitivity $100 \%$, specificity $65 \%$ and $69.6 \%$ of patients correctly classified for a cut-off point $\geq 19.5$. On the other hand, C3 and C4 also had a high discrimination capacity for flare (AUC 0.804 and 0.837 respectively). Comparing the three parameters, C4 achieved the highest AUC (0.837, 95\% Cl: 0.663-1000).

Conclusions: Lambda free light chains have a good discrimination capacity for SLE flares and could be useful as a SLE flare biomarker. Longitudinal studies with a larger number of patients are necessary to evaluate its usefulness as a flare predictor.

Disclosure of Interest: None declared

DOI: 10.1136/annrheumdis-2018-eular.5374

\section{FRI0345 ASSOCIATION BETWEEN CIGARETTE SMOKING AND SYSTEMIC LUPUS ERYTHEMATOSUS (SLE) - AN UPDATED META-ANALYSIS OF CASE-CONTROL AND COHORT STUDIES}

M.H.Y. Chua, I. A. T. Ng, A. Mak. Medicine, National University of Singapore, Singapore, Singapore

Background: While the association between cigarette smoking and the occurrence of SLE has been studied over the past two decades, conflicting results have rendered such association controversial. With the global increase in woman smokers and more prospective data addressing the risk of the development of SLE and cigarette smoking being available, it is timely to provide an update on the evidence of the relationship between cigarette smoking and the occurrence of SLE.
Objectives: To study the association between cigarette smoking and the odds, as well as the risk of SLE occurrence by meta-analyses of case-control and cohort studies.

Methods: We performed an extensive literature search using the relevant keywords including "systemic lupus erythematosus"; "lupus'; "smoking"; "cigarette smoking"; "environmental"; "autoimmune disease" and "connective tissue disease" in various combinations to identify case-control and cohort studies address ing the relationship between cigarette smoking and SLE published in English in computerised databases including PubMed (from 1966 to , Jan 2018 Embase (1980 to Jan 2018 and Cochrane Central Register of Control Trials (last quarter of 2017). Effect sizes were pooled as odds ratio (OR) and relative risk (RR) and the corresponding $95 \% \mathrm{Cl}$ for case-control and cohort studies, respectively. Heterogeneity was studied by $I^{2}$. If the effect sizes were associated with low heterogeneity $\left(I^{2}<40\right)$, the fixed effects model was used. Otherwise $\left(I^{2} \geq 40\right)$, the random effects model was used, based on the model suggested by DerSimonian and Laird.

Results: Thirteen relevant studies (10 retrospective case-control and 3 cohort) were selected for these meta-analyses. Data were aggregated based on smoking statuses comprising current, past, ever and never smoking. Analyses of case-control studies revealed significant relationships between the occurrence of SLE and current smoking (OR 1.639 [1.171-2.295], $\mathrm{p}=0.004, \mathrm{I}^{2}=85.8$ ), and ever smoking (OR 1.438 [1.037-1.995], $\mathrm{p}=0.029, \mathrm{I}^{2}=86.3$ ). However, no significant relationship was found between the occurrence of SLE and past smoking (OR 1.208 [0.8531.711], $\left.p=\left.0.286\right|^{2}=74.7\right)$. While no relationship was found between the development of SLE and current smoking (RR 1.002 [0.680-1.475], $p=0.994, \mathrm{I}^{2}=51.6$ ), and ever smoking (RR 1.163 [0.944-1.433], $\left.p=0.157, \mathrm{I}^{2}=0\right)$ when the effect sizes of cohort studies were amalgamated, a significant risk in the development of SLE was found amongst ex-smokers compared with never smokers when the prospec tive data were combined (RR 1.351 [1.085-1.681], $p=0.007, I^{2}=28.0$ ).

Conclusions: Smoking is associated with the occurrence of SLE based on the aggregation of effect sizes from case-control and cohort studies. The higher risk of development of SLE in ex-smokers but not among current and ever smokers compared to never smokers based on prospective data warrants further mechanistic studies to unravel the actual immunologic impact of cigarette smoke on SLE development in different subsets of SLE patients.

Disclosure of Interest: None declared DOI: 10.1136/annrheumdis-2018-eular.6836

\section{FRI0346 \\ NEUTROPENIA IN SYSTEMIC LUPUS: PREVALENCE, SPECIFIC FEATURES AND CLINICAL CONSEQUENCES. RESULTS FROM THE LARGE UPPER RHINE DATABASE LBBR}

A. Meyer ${ }^{1}$, P.-E. Gavand ${ }^{1}$, G. Blaison ${ }^{2}$, T. Martin $^{1}$, A.-S. Korganow ${ }^{1}$, A. Guffroy ${ }^{1}$, on behalf of LBBR Study Group. ${ }^{1}$ Clinical Immunology, CHRU Strasbourg, Strasbourg; ${ }^{2}$ Internal Medicine, $\mathrm{CH}$ Colmar, Colmar, France

Background: The prevalence, pathophysiology and underlying causes or consequences of neutropenia in systemic lupus erythematosus (SLE) are still not well defined even if neutropenia seems to be rather common in this disease. ${ }^{1}$

Objectives: To evaluate the prevalence of neutropenia in a large cohort of SLE patients and to identify correlation between neutropenia and other socio-demographical, clinical, serological or therapeutic factors.

To precise the influence of chronicity or severity of neutropenia in the course of SLE.

Methods: We used the LBBR database, a cross-sectional collection of detailed socio-demographic, clinical, serological and therapeutic data from 1,078 SLE patients (14 french or german Upper Rhine Hospitals).

Neutropenia was defined by the presence of less than 1800 circulating neutrophils $\times 10^{9} / \mathrm{L}$. Patients with and without neutropenia were compared considering 47 variables.

The second part of the study focused on a subgroup of SLE LBBR patients for which full data were available about the duration and depth of neutropenia Chronic neutropenia was defined by neutrophils count less than $1,500 \times 10^{9} / \mathrm{L}$ during at least 6 months and moderate and severe neutropenias were defined by neutrophils count less than $1,000 \times 10^{9} / \mathrm{L}$

Results: Among 1078 SLE patients, 223 (20.7\%) were registered with neutropenia during their history. Mean age and sex ratio were comparable to the whole SLE cohort and to patients without neutropenia (mean age: 43.9 years old, sex ratio: $194 \mathrm{~F} / 28 \mathrm{M}$ ). In multivariate analysis, neutropenia was associated with lymphopenia $(\mathrm{OR}=3.44(2.48-4.80), \mathrm{p}=0.0002)$ and thrombopenia $(\mathrm{OR}=3.59(2.55$ $5.06), p=0.0002)$. There was no association with susceptibility to infections (OR $0.97(0.52-1.80), p=0.6640)$, neither with SLEDAI score, SLE treatments or other ACR criteria. 